\title{
Validade comercial e aceitabilidade da carne de tartaruga-da-amazônia (P.expansa)
}

\author{
Arlene GASPAR ${ }^{1}$, Teófilo José Pimentel da SILVA² \\ RESUMO \\ Esse experimento foi elaborado em parceria com o CENAQUA (Centro Nacional de Quelônios do Amazonas) e a Pró-Fauna \\ (localizada em Iguape - SP) objetivando o estudo da validade comercial e a aceitabilidade da carne de tartaruga-da-amazônia \\ criada em cativeiro. Foi avaliado o efeito do tempo de estocagem de amostras de carne de 14 tartarugas (7 fêmeas e 7 machos) \\ nos dias $01,30,60$ e 120, estocadas a $-10{ }^{\circ} \mathrm{C}$, nos valores de $\mathrm{pH}$ e de Bases Voláteis Totais (BVT), cor, textura, odor e prova \\ de cocção. Observou-se diferença significativa entre os valores de $\mathrm{pH}$ em relação ao tempo de estocagem nos dias 01 (5,81 \\ e 5,81), 30 (6,04 e 6,05), 60 (6,22 e 6,23) e 120 (6,30 e 6,30), respectivamente para a carne das fêmeas e machos. Entre os \\ sexos não houve diferença significativa. Quanto a BVT o tempo de estocagem influenciou significativamente entre os dias 01 \\ (11,34 e 11,74 g/100g), 30 (13,92 e 13,90 g/100g) e 120 (17,62 e 17,82 g/100g); entre os dias $30(13,92$ e 13,90 g/100g) \\ e $60(14,02$ e 14,06 g/100g) não observou-se diferença significativa, respectivamente para a carne de fêmeas e machos. Entre \\ os sexos não ocorreu diferenças significativas. A cor, textura, odor e a prova de cocção não apresentaram alteração ao longo \\ do tempo de estocagem. A carne de tartaruga obteve media de aceitabilidade global de 7,04. De acordo com os resultados \\ obtidos concluímos que, pelas provas de $\mathrm{pH}$ e BVT, a carne de tartaruga pode ter uma validade comercial de 120 dias, quando \\ embaladas a vácuo e estocadas a $-10^{\circ} \mathrm{C}$, e que a os provadores gostaram muito desse tipo de carne.
}

PALAVRAS-CHAVE: animais silvestres; qualidade, estocagem.

\section{Shelf-life and acceptance of Amazon turtle meat (P.expansa)}

\section{ABSTRACT}

This research was developed in partnership with CENAQUA and Pró-Fauna with the objective of studying commercial validity and acceptance of the meat of Amazon-turtle that was bred in captivity. The effect of the storage period was evaluated on meat samples of fourteen turtles ( 7 female and 7 male ) stored in a period of $1,30,60$, and 120 days at a temperature of -100 Celsius, concerning to values of $\mathrm{pH}$, total volatile bases, color, scent, and cooking test. Significant difference was observed in relation to $\mathrm{pH}$ values and the period of storage as follow: in the period of 1 (5.81 and 5.81), 30 (6.04 and 6.05), 60 (6.22 and 6.23), and 120 (6.30 and 6.30) days, respectively either for the meat of female turtle or male. There was not significant difference concerning to sex. As for total volatile bases (TVB), the period of storage has influenced significantly: in the period of $1(11.34$ and $11.74 \mathrm{~g} / 100 \mathrm{~g}), 30(13.92$ and $13.90 \mathrm{~g} / 100 \mathrm{~g})$, and $120(17.62$ and $17.82 \mathrm{~g} / 100 \mathrm{~g})$; in the period of $30(13.92$ and $13.90 \mathrm{~g} / 100 \mathrm{~g})$ and $60(14.02$ and $14.06 \mathrm{~g} / 100 \mathrm{~g})$ days, significant difference was not observed, respectively for female and male meat. Not significant difference occurred in relation to sex. The result for color, texture, scent, and cooking test did not present any alteration along the period of storage. The meat of turtle obtained a 7.04 average of general acceptability that shows the acceptance of the tasters for this sort of meat. According to the results for $\mathrm{pH}$ and TVB, we have concluded that this meat of turtle may have 120 days of commercial validity once it is vacuum-packed and stored at -100Celsius, and that it presents good acceptance for the tasters.

KEY WORDS: wild animal, quality, storage.

\footnotetext{
1 Universidade Federal Rural do Rio de Janeiro. E-mail: arlene@ufrri.br

2 Universidade Federal Fluminense. E-mail: teofilo.silva@pq.cnpq.br
} 


\section{INTRODUÇÃO}

Com o crescimento da população e o interesse em preservar o meio ambiente torna-se necessário buscar fontes protéicas alternativas e conhecer o comportamento da qualidade de carnes de animais silvestres. Entretanto, o uso dessas espécies deve ser racional, adequado, com mecanismos de exploração sistemática e científica em que seja priorizada a preservação e a sustentabilidade dos sistemas.

A fauna brasileira constitui uma fonte importante de alimentos. Diversas espécies como aves, pacas, cervos, capivaras, tatus, tartarugas, etc., são citadas como fonte protéica para as populaçôes rurais e com demanda crescente nos grandes centros urbanos. Apesar do consumo desse tipo de carne ser elevado, não existe estudo sobre a vida-de-prateleira da mesma.

O pescado é uma das principais fontes de proteínas na alimentação humana. Além de ser um bom alimento, dele também são produzidos óleos, raçōes e produtos de valor para a indústria. Essa diversidade de utilizações ocorre devido à existência de diversas espécies de peixes e variadas estruturas histológicas e composição química de suas partes (PEREDA et. al., 2005).

Segundo o Regulamento de Inspeção Industrial e Sanitária de Produtos de Origem Animal - RIISPOA, art. 438 (Brasil, 1997), como carne de "PESCADO" compreende as carnes de peixes, crustáceos, moluscos, anfíbios, quelônios e mamíferos de água doce ou salgada, usados na alimentação humana.

A qualidade dos alimentos, do ponto de vista da validade comercial, é definida por parâmetros fisiológicos, valores nutricionais e atributos sensoriais como cor, sabor e textura ou consistência. A diminuição da qualidade e a redução da validade comercial pode ser consequiência do efeito de uma ou mais destas propriedades (PFEIFFER ET. AL., 1999).

$\mathrm{O}$ estudo da validade comercial de novos produtos alimentícios consiste em determinar o prazo de validade que deverá ser estampado nas embalagens. Esse período, em alimentos resfriados, é amplamente dependente do nível de contaminação inicial e o crescimento microbiano é o principal fator que determina sua vida útil. Por outro lado, durante a estocagem em condiçōes de congelamento, a oxidação lipídica e as mudanças associadas a ela são as maiores causas da perda de qualidade das carnes de alimentos que contenham gorduras insaturadas (GRAY \& MONAHAN, 1992). A oxidação dos ácidos graxos insaturados ocorre por meio da formação de radicais livres com posterior ataque à cadeia insaturada, envolvendo fases de iniciação, propagação e término (FARMER \& SUTON, 1943).

A carne quando imprópria ao consumo apresenta mudanças como: limosidade superficial; produção de gás; oxidação de gorduras resultando em off-flavour, mudança na cor com produção de pigmentos de coloração diversa; perda de textura; etc. (BOARD, 1983).

O processo de deterioração do pescado ocorre por mudanças nas propriedades físicas e químicas dos músculos, durante armazenamento refrigerado, por degradação de ATP, produção de compostos voláteis e outras substâncias. A quantidade de substâncias nitrogenadas disponíveis nos músculos na forma de aminoácidos livres e peptídeos simples exercem papel importante no aparecimento de outros compostos de degradação.

A avaliação do estado de frescor do pescado pode ser realizada por métodos sensoriais, físicos e químicos. $\mathrm{O}$ pH é considerado por alguns autores como um índice seguro de medida do estado de frescor ou do início da deteriora. Outro parâmetro muito utilizado é a determinação das Bases voláteis Totais (BVT). As BVTs compreendem compostos como amônia, trimetilamina e dimetilamina. No início da deteriora a BVT mais encontrada é a amônia originária da degradação do ATP. O teor de BVT para peixes de excelente estado de frescor varia de 5 a $10 \mathrm{mg} / 100 \mathrm{~g}$ de carne, em estado de frescor razoável variam de 15 a $25 \mathrm{mg} / 100 \mathrm{~g}$, no início da putrefação estão na faixa de $30 \mathrm{a} 40 \mathrm{mg} / 100 \mathrm{~g}$ e em adiantado estado de decomposição estão acima de $50 \mathrm{mg} / 100 \mathrm{~g}$ (OGAWA \& MAIA, 1999).

O RIISPOA art. 443 (Brasil, 1997) classifica pescado fresco quando: a) a reação de gás sulfrídrico é negativa; b) o $\mathrm{pH}$ de carne externa é inferior a 6,8 e o da carne interna é inferior a 6,5, para peixes; e, c) quando as bases voláteis totais inferiores (BVT) são inferiores a $30 \mathrm{mg}$ de nitrogênio (processo de difusão) por $100 \mathrm{~g}$ de carnes.

Segundo Chaves (2001) a análise sensorial é utilizada para evocar, medir, analisar, e interpretar as reaçōes e as características de alimentos e outros produtos de consumo, da forma como são percebidas pelos sentidos da visão, olfato, gosto, tato e audição.

Normalmente, os métodos sensoriais são utilizados para identificar as características ou propriedades de interesse na qualidade sensorial do alimento (JACKSON, 1985). Em métodos baseados na aceitação do mercado consumidor, os provadores são escolhidos ao acaso e as decisōes de aceitação e ou preferência são obtidas.

O teste de aceitação é utilizado quando se deseja conhecer o comportamento afetivo do consumidor com relação ao produto (MEILGAARD; CIVILLE; CARR, 1991). Os testes de aceitação requerem um grande número de participantes (acima de 40 ou 50) que representem à população de consumidores atuais ou potenciais do produto.

Segundo Chaves (2001), a escala hedônica pode ser utilizada em testes de aceitação em laboratórios e tem por objetivo obter 
informações sobre a provável aceitação de produtos pelo consumidor nas fases iniciais de desenvolvimento.

Nesse estudo, objetivou-se estudar a validade comercial de amostras de carne de tartaruga-da-amazônia, estocadas a $-10^{\circ} \mathrm{C}$ nos dias $01,30,60$ e 120 através da cor, textura, odor, prova de cocção, e com base nos valores de determinação do pH e das Bases Voláteis Totais (BVT) e a aceitabilidade dessa matéria prima.

\section{MATERIAL E MÉTODOS}

Utilizou-se 14 tartarugas (07 fêmeas e 07 machos), com idade aproximada de 2 a 3 anos, peso médio de 1,500 a $2,500 \mathrm{~kg}$, escolhidas aleatoriamente, oriundas de criatórios registrados no IBAMA, localizados em Diorama - Goiás. Os animais foram criados em lagoas artificiais, alimentadas à base de vegetais e legumes.

As tartarugas foram pescadas, lacrados, acomodados em gaiolas de metal e transportados em caminhōes para a fazenda Devaneio localizada em Iguape-SP, de propriedade da empresa Pró-Fauna, onde localiza-se um abatedouro de animais silvestres registrado no IBAMA e no MAPA. Durante o transporte, os animais foram molhados diversas vezes com o objetivo de mantê-los o mais confortável possível, minimizando com isso o estresse provocado pelo transporte. Após o abate e desossa, a carne foi congelada a temperatura de $-10^{\circ} \mathrm{C}$, acondicionada em embalagem isotérmica e levadas ao Departamento de Tecnologia de Alimentos da Universidade Federal Rural do Rio de Janeiro (DTA/UFRRJ) onde foram realizadas as análises físicas e químicas.

Avaliou-se o tempo de estocagem nos dias 01, 30, 60 e 120, através da cor, textura, odor, prova de cocção, determinação do pH e das Bases Voláteis Totais (BVT) (Ellis, M.J, 1996; Brasil, 1999).

Foram realizados testes de aceitação sensorial (teste afetivo de aceitação) utilizando-se uma equipe de 50 provadores de ambos os sexos, com idade variando de 20 a 60 anos, consumidores usuais de carne, porém sem nunca terem consumido carne de tartaruga. As amostras foram temperadas com sal e preparadas em molho de tomate, e oferecidas aos provadores apresentadas no formato de cubos $(1 \times 1 \mathrm{~cm})$, em pratinhos brancos de plásticos codificados por letras, contendo porções de $20 \mathrm{~g}$ cada, e em temperatura média de $50^{\circ} \mathrm{C}$. No momento da avaliação sensorial de aceitabilidade, os provadores receberam uma ficha contendo uma escala de pontos variando de 1 a 9 , solicitando que fosse avaliado o quanto gostaram ou desgostaram da amostra servida, por meio de uma escala hedônica estruturada mista de 9 pontos $(9$ gostei muitíssimo; 8- gostei muito; 7 - gostei moderadamente; 6 - gostei ligeiramente; 5 - não gostei nem desgostei; 4 - desgostei ligeiramente; 3 - desgostei moderadamente; 2 - desgostei muito e 1 - desgostei muitíssimo). A aplicação dos testes sensoriais seguiu método descrito por Stone, H. e Sidel, J. L. (1993). Nos resultados obtidos aplicou-se a ANOVA fator único para avaliar se havia diferenças entre as médias obtidas, posteriormente aplicou-se o teste de Tukey, os resultados foram expressos por histograma de barras.

Os resultados obtidos nas análises fisico-químicas, onde se queria avaliar a se havia interferência do sexo em relação ao tempo de estocagem, foi utilizado um delineamento fatorial $2^{2}$ (sexo x tempo de estocagem), segundo Gomes (1987) e o Instituto de Tecnologia de Alimentos - ITAL (1982). Quando havia diferenças significativas classificava-se pelo teste de Tukey. Os cálculos da análise estatística foram realizados utilizando-se um programa estatístico desenvolvido pela Universidade Estadual Paulista - UNESP em Jaboticabal.

\section{RESULTADOS E DISCUSSÃO}

Quanto aos parâmetros cor, textura, odor e prova de cocção apresentaram-se característicos durante o período de estocagem. A carne estocada não apresentou off-flavor, alteração de cor, textura e limosidade superficial, atendendo assim ao art. 443 do RIISPOA (Brasil, 1997).

$\mathrm{Na}$ Tabela 1 está apresentado os resultados médios de $\mathrm{pH}$ da carne de tartarugas fêmeas e machos pelo período de 120 dias estocadas a $-10^{\circ} \mathrm{C}$.

Tabela 1 - Valores médios do pH e desvio padrão obtidos na carne de tartarugas (fêmeas e machos) em relação ao tempo de estocagem a $-10^{\circ} \mathrm{C}$.

\begin{tabular}{lcc}
\hline \multirow{2}{*}{$\begin{array}{l}\text { Tempo de estocagem } \\
\text { (em dias) }\end{array}$} & \multicolumn{2}{c}{$\mathrm{pH}$} \\
\cline { 2 - 3 } & Fêmea & Macho \\
\hline 01 & $5,81 \pm 0,05^{\mathrm{a}}$ & $5,81 \pm 0,05^{\mathrm{a}}$ \\
30 & $6,04 \pm 0,02^{\mathrm{b}}$ & $6,05 \pm 0,02^{\mathrm{b}}$ \\
60 & $6,22 \pm 0,03^{\mathrm{c}}$ & $6,23 \pm 0,03^{\mathrm{c}}$ \\
120 & $6,30 \pm 0,03^{\mathrm{d}}$ & $6,30 \pm 0,08^{\mathrm{d}}$ \\
\hline
\end{tabular}

Os resultados são médias de três determinações.

Letras iguais entre colunas demonstram que não há diferenças significativas entre os sexos.

Letras diferentes entre linhas demonstram diferença significativa $(P<0,01)$ em relação ao tempo de estocagem.

Quanto ao $\mathrm{pH}$, não foi observada diferença significativa entre a carne de fêmeas e machos. Entretanto, quanto aos dias de estocagem, observou-se diferenças significativas $(P<0,01)$. Verificou-se que houve um aumento crescente em relação aos valores de $\mathrm{pH}$ ao longo do tempo de estocagem para as carnes de fêmeas e machos, entretanto esses valores apresentaram-se abaixo do máximo exigido pela legislação que é de 6,5. Assim, com base nos valores médios de $\mathrm{pH}$, para as amostras de carne de tartaruga congeladas a $-10^{\circ} \mathrm{C}$ logo após os processos de abate e mantidas a essa temperatura, é possível propor períodos de prazos de validade de 120 dias de estocagem. As variaçôes 
dos valores médios de $\mathrm{pH}$ nos diferentes tempos de estocagem podem ser observadas na Figura 1.

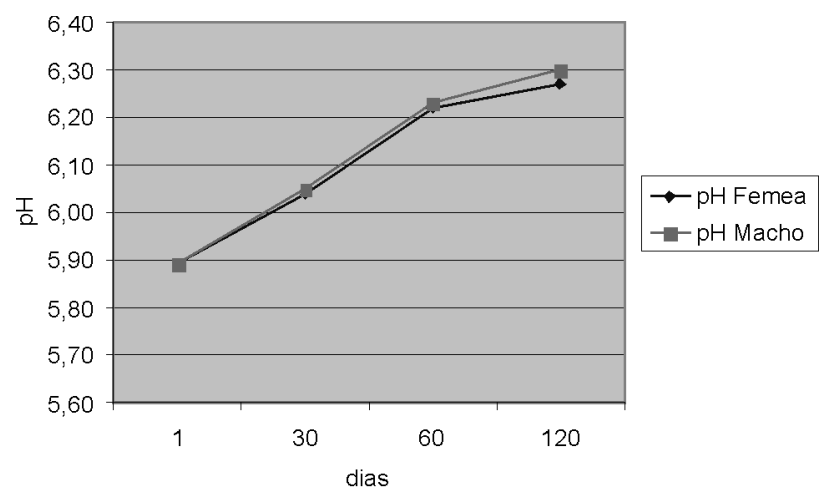

Figura 1 - Representação gráfica do valor do $\mathrm{pH}$ da carne de tartarugas (fêmeas e machos) em relação ao tempo de estocagem a $-10^{\circ} \mathrm{C}$.

A Tabela 2 apresenta os valores médios de BVT determinados na carne de tartarugas fêmeas e machos, estocadas por 120 dias a $-10{ }^{\circ} \mathrm{C}$.

Tabela 2 - Valores de BVT da carne de tartarugas (fêmeas e machos) em relação ao tempo de estocagem $\mathrm{a}-10^{\circ} \mathrm{C}$.

\begin{tabular}{lcc}
\hline \multirow{2}{*}{$\begin{array}{l}\text { Tempo de estocagem } \\
\text { (em dias) }\end{array}$} & \multicolumn{2}{c}{ BVT $(\mathrm{mg} / 100 \mathrm{~g})$} \\
\cline { 2 - 3 } & Fêmea & Macho \\
\hline 01 & $11,34 \pm 0,32^{\mathrm{a}}$ & $11,74 \pm 0,41^{\mathrm{a}}$ \\
30 & $13,92 \pm 0,05^{\mathrm{b}}$ & $13,90 \pm 0,07^{\mathrm{b}}$ \\
60 & $14,02 \pm 0,01^{\mathrm{b}}$ & $14,06 \pm 0,03^{\mathrm{b}}$ \\
120 & $17,82 \pm 0,13^{\mathrm{c}}$ & $17,62 \pm 0,86^{\mathrm{c}}$ \\
\hline
\end{tabular}

Os resultados são médias de três determinações.

Letras iguais entre colunas demonstram que não há diferenças significativas entre os sexos.

Letras diferentes entre linhas demonstram diferença significativa $(P<0,01)$ em relação ao tempo de estocagem.

Podemos observar que os resultados obtidos não apresentaram-se significativamente diferente entre os sexos. Quanto ao tempo de estocagem, os valores apresentaram diferenças significativas entre os dias 01,30 e 120 . Entre 30 e 60 dias não foram observadas diferenças significativas. Esses resultados indicam estado de fresco das amostras, pois o limite exigido pela legislação é de no máximo $30 \mathrm{mg} / 100 \mathrm{~g}$ (Brasil, 1997). Na Figura 2 é possível observar a evolução crescente dos teores de BVT durante os 120 dias de estocagem.

A carne de tartaruga obteve média global de aceitabilidade de 7,04 $( \pm 1,48)$, demonstrando que os provadores gostaram desse tipo de carne. A Figura 3 apresenta o percentual de provadores e os escores afetivos dados à carne de tartaruga por provadores não treinados durante o teste de aceitação sensorial. De acordo com os histogramas de frequiência das notas sensoriais, pôde-se verificar que apenas $2 \%$ dos provadores desgostaram ligeiramente da carne e $18 \%$ foram indiferentes,
$22 \%$ gostaram ligeiramente, $10 \%$ gostaram moderadamente, $28 \%$ gostaram muito e $20 \%$ gostaram muitíssimo.

Podemos observar que apesar dos provadores nunca terem comido carne de tartaruga, a mesma obteve uma boa aceitação. Esses dados estão concordantes com os dados obtidos em pesquisa realizada por Ferreira e Araújo (1979), onde a maior demanda de consumo foi para carne de tartaruga de água doce, pois era o prato mais popular entre o público em geral.

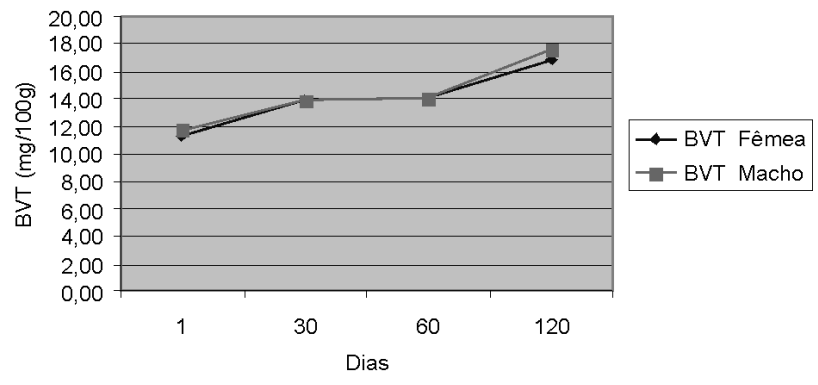

Figura 2 - Valor do teor de BVT da carne de tartarugas (fêmeas e machos) em relação ao tempo de estocagem $\mathrm{a}-10^{\circ} \mathrm{C}$.

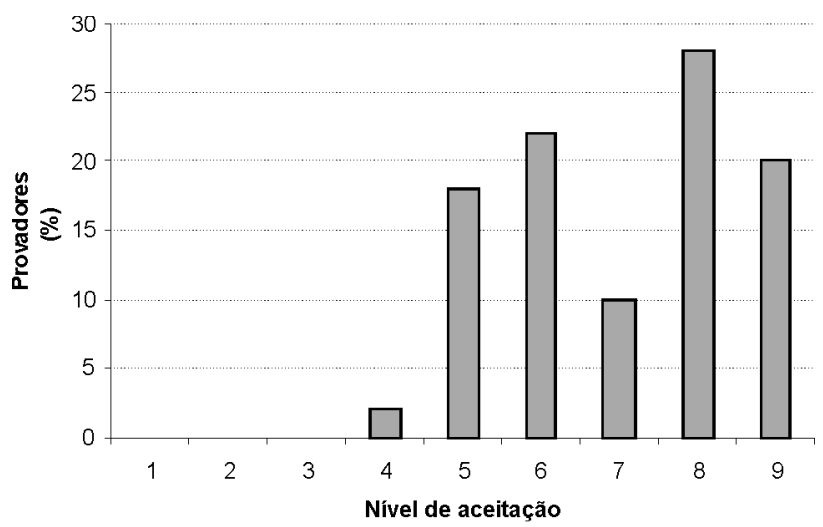

Figura 3 - Percentual de pessoas e os escores afetivos dados à carne de tartaruga por provadores não treinados durante 0 teste de aceitação sensorial.

\section{CONCLUSÃO}

De acordo com os resultados obtidos nessa pesquisa é possível concluir que, pelas provas de $\mathrm{pH}$ e BVT, a carne de tartaruga pode ser mantida em boa conservação por até 120 dias, quando embaladas a vácuo e estocadas a $-10^{\circ} \mathrm{C}$, e a carne de tartaruga apresentou boa aceitabilidade entre o grupo de provadores.

\section{BIBLIOGRAFIA CITADA}

BOARD, R. G. 1983 A modern introduction to food microbiology. v.8, Bath - England: Blackwell, 230p.

BRASIL, 1999 INSTRUÇÃO NORMATIVA No 20, de 21/07/1999. Métodos analíticos físico-químicos para controle de produtos cárneos e seus ingredientes - sal e salmoura. Ministério da Agricultura. Secretaria Nacional de Defesa Agropecuária. Brasília. 
BRASIL, 1997 Ministério da Agricultura, do Abastecimento Secretaria Nacional de defesa Agropecuária. Regulamento da Inspeção Industrial e Sanitária de Produtos de Origem Animal (Aprovado pelo Decreto no 30.691, de 29-03-52, alterado pelos Decretos $n^{\circ}$ s 1.255 de 25-06-62, 1.236 de 02-09-94, no 1.812 de 08-02-96 e no 2.244 de 05-06-97). DIPOA - MAPA, Brasília - DF, 241p.

CHAVES, J. B. P. Métodos de diferença em Avaliação Sensorial de Alimentos e Bebidas. 2001. Cadernos Didáticos, 33. Viçosa: UFV. 91p.

ELLIS, M.J. 1996. Shelf life evaluation of foods. London: Black Academic \& Professional, 321p.

JACKSON, H.W. Oil flavors quality assessment. 1985. In: APPLEWHITE, T.H. Bailey's industrial oil and fat products. New York: John Wiley, v. 3, p.243-272.

FARMER, E. H.; SUTON, D.A. 1943. The course of the autoxidation reactions in polyisoprenes and allied compounds. Part IV. The isolation and construction of photo chemicallyformed methyl oleate peroxide. Journal of Chemical Society, p. 119.

FERREIRA, M.; ARAÚJO, V. C. 1970. Espécies da Fauna Amazônica potencialmente preferidas para Consumo nos Restaurantes de Manaus. Brasil Florestal, v.7, n.25, p. 59-69.
GRAY, J. I.; MONAHAN, F. J. 1992. Measurement of lipid oxidation in meat and meat products. Trends Food Science and Technology., v. 3, n.12, p. 315-319.

MEILGAARD, M.; CIVILLE, G.V.; CARR, B.T. 1991. Sensory evaluation techniques. 2 ed. Florida, USA: CRC press, 354p.

OGAWA, M.; MAIA, E. L. 1999. Manual de pesca. Ciência e tecnologia do pescado. V. I, São Paulo: Ed. Varela, 430p.

PEREDA, J. A. O. et. al. TENOLOGIA DE ALIMENTOS: Alimentos de Origem Animal. 2005. Vol. 2. Editora Artmed. São Paulo, $234 \mathrm{p}$.

PFEIFFER C., D'AUJOURD'HUI, J.W., NUESSLI, J., ESCHER, F. 1999. Optimizing food packaging and shelf life. Food Technol., Chicago, v.53, n.6, p.52-59.

STONE, H., SIDEL, J. 1993. Sensory evaluation practices. Academic Press: New York. 3 ed., p.338.

Recebido em 29/11/2007

Aceito em 28/05/2009 
